\title{
ON SEMISIMPLE SEMIGROUP RINGS
}

\author{
MARK L. TEPLY, ${ }^{1}$ E. GEIS TURMAN AND ANTONIO QUESADA
}

\begin{abstract}
Let $\pi$ be a property of rings that satisfies the conditions that (i) homomorphic images of $\pi$-rings are $\pi$-rings and (ii) ideals of $\pi$-rings are $\pi$-rings. Let $S$ be a semilattice $P$ of semigroups $S_{\alpha}$. If each semigroup ring $R\left[S_{\alpha}\right](\alpha \in P)$ is $\pi$-semisimple, then the semigroup ring $R\left[S_{\alpha}\right]$ is also $\pi$-semisimple. Conditions are found on $P$ to insure that each $R\left[S_{\alpha}\right](\alpha \in P)$ is $\pi$-semisimple whenever $S$ is a strong semilattice $P$ of semigroups $S_{\alpha}$ and $R[S]$ is $\pi$-semisimple. Examples are given to show that the conditions on $P$ cannot be removed. These results and examples answer several questions raised by J. Weissglass.
\end{abstract}

Let $\pi$ be a property of rings. A ring is called a $\pi$-ring if it has property $\pi$. An ideal $I$ of a ring is a $\pi$-ideal if $I$ is a $\pi$-ring. A ring is $\pi$-semisimple if it has no nonzero $\pi$-ideals. Henceforth, we assume that the property $\pi$ satisfies the conditions, (i) homomorphic images of $\pi$-rings are $\pi$-rings and (ii) ideals of $\pi$-rings are $\pi$-rings. For example, the properties of being nil, nilpotent, or left quasi-regular are such properties.

Let $R$ be a ring and let $S$ be a semigroup. Then the semigroup ring $R[S]$ consists of all formal sums $\sum_{s \in s_{s}} r_{s}$ such that $r_{s} \in R$ and $r_{s}=0$ for all but finitely many $s \in S$; addition and multiplication are defined in the obvious manner (see [1], [2], [3], [6], [7]). Several authors have studied properties $\pi$ for specialized semigroup rings. For example, Gilmer and Parker [2] compute the nil radical of $R[S]$ under the assumption that $R[S]$ is commutative. Passman [4] discusses many radical properties under the assumption that $R$ is a field and $S$ is a group. Krempa [3] computes the lower Baer radical of $R[S]$ when $S$ is a cancellative $\Omega_{1}$-semigroup. Quesada [6] also computes the lower Baer radical of $R[S]$ in several interesting cases.

Let $P$ be a semilattice whose natural order is indicated by $>$. As in [5], a semigroup $S$ is called a strong semilattice $P$ of semigroups $S_{\alpha}$ if there exists a family of homomorphisms $\varphi_{\alpha, \beta}: S_{\alpha} \rightarrow S_{\beta}(\alpha, \beta \in P ; \alpha \geqslant \beta)$ satisfying the following conditions.

(a) $S$ is the disjoint union of the semigroups $S_{\alpha}$.

(b) $\varphi_{\alpha, \alpha}$ is the identity map $S_{\alpha}$.

(c) If $\alpha>\beta>\gamma$, then $\varphi_{\alpha, \beta} \varphi_{\beta, \gamma}=\varphi_{\alpha, \gamma}$.

(d) If $a \in S_{\alpha}$ and $b \in S_{\beta}$, then multiplication in $S$ is determined by $a \cdot b=$ $\left(a \varphi_{\alpha, \alpha \beta}\right) \cdot\left(b \varphi_{\beta, \alpha \beta}\right) \in S_{\alpha \beta}$.

Received by the editors November 14, 1978 and, in revised form, February 16, 1979.

AMS (MOS) subject classifications (1970). Primary 20M25; Secondary 16A12, 16A20, 16A21.

Key words and phrases. Semigroup ring, semilattice, $\pi$-semisimple.

${ }^{1}$ This author received support from NSF grant MCS 77-01818.

() 1980 American Mathematical Society 0002-9939/80/0000-0250/\$02.75 
For more details, see $\S$ III.7 of [5]. We note that each $\varphi_{\alpha, \beta}$ has a natural extension to a ring homomorphism from $R\left[S_{\alpha}\right]$ to $R\left[S_{\beta}\right]$ :

$$
\sum_{s \in S_{\alpha}} r_{s} s \rightarrow \sum_{s \in S_{\alpha}} r_{s}\left(s \varphi_{\alpha, \beta}\right)
$$

We also denote this extension by $\varphi_{\alpha, \beta}$ for convenience. For the rest of this paper, $S$ will denote a strong semilattice $P$ of semigroups $S_{\alpha}$.

The purpose of this paper is to study conditions relating the $\pi$-semisimplicity of $R[S]$ to the $\pi$-semisimplicity of the $R\left[S_{\alpha}\right](\alpha \in P)$. If each $R\left[S_{\alpha}\right]$ is $\pi$-semisimple, then $R[S]$ is $\pi$-semisimple. This result is an immediate consequence of Theorem 1 , which generalizes [6, Theorem 4.21] and [7, Theorem 1 and Corollary 1] and which answers affirmatively Question 4 of Weissglass [7, p. 477]. However, if $R[S]$ is $\pi$-semisimple, not all of the $R\left[S_{\alpha}\right]$ need be $\pi$-semisimple. A problem may occur if $R\left[S_{\alpha}\right]$ has a nonzero $\pi$-ideal and there are infinitely many $\beta \in P$ closely beneath $\alpha$ in the order $>$ of $P$. We determine conditions on $P$ in our main result (Theorem 2) which guarantee that $R[S]$ is $\pi$-semisimple if and only if $R\left[S_{\alpha}\right]$ is $\pi$-semisimple for all $\alpha \in P$. The key part of the proof of Theorem 2 is the construction of a special type of ideal of $R[S]$ from an ideal of $R\left[S_{\alpha}\right]$; this construction is done in Lemma 2. Theorem 2 gives an answer to Question 1 of Wiessglass [7, p. 477] for a semigroup ring case. Examples are given to show that the conditions on $P$ in the hypothesis of Theorem 2 cannot be dropped when $\pi$ is the property of being nil, nilpotent, or left quasi-regular, even if each $S_{\alpha}$ is a group and $R$ is a field. Thus these examples answer in the negative Question 7 of Weissglass [7, p. 477]. A modification of these examples is also used to answer in the negative Question 2 of Weissglass [7, p. 477] concerning the Jacobson radical of certain commutative rings.

Many interesting semigroups appear as strong semilattices $P$ of semigroups $S_{\alpha}$, where $S_{\alpha}$ is a special type of semigroup; for example, see [1, Theorem 4.11] and [5, Theorem IV.4.3 and Corollaries IV.4.6, IV.4.7]. Since conditions for many special types of semigroup rings to be $\pi$-semisimple are known, our theorems often provide a realistic test for the $\pi$-semisimplicity of $R[S]$.

Before stating our first result, we need a generalization of a semigroup ring $R\left[S^{\prime}\right]$, where $S^{\prime}$ is any semilattice of semigroups.

In [7], a ring $T$ is called a supplementary semilattice sum of subrings $T_{\alpha}(\alpha \in P)$ if the following conditions hold: $T=\sum_{\alpha \in P} T_{\alpha}, T_{\alpha} T_{\beta} \subseteq T_{\alpha \beta}$ for all $\alpha, \beta \in P$, and $T_{\alpha} \cap\left(\Sigma_{\alpha \neq \beta} T_{\beta}\right)=0$ for each $\alpha \in P$. Clearly, $R[S]$ is always a supplementary semilattice sum of subrings with $T_{\alpha}=R\left[S_{\alpha}\right]$. If $t \in T$, we define

$$
P \text {-supp } t=\left\{\alpha \in P \mid t=\sum_{\alpha \in P} t_{\alpha} \text { and } t_{\alpha} \neq 0\right\}
$$

We are now ready to generalize [6, Theorem 4.21] and [7, Theorem 1].

THEOREM 1. Let $T=\Sigma_{\alpha \in P} T_{\alpha}$ be a supplementary semilattice sum of subrings. If $T_{\alpha}$ is $\pi$-semisimple for each $\alpha \in P$, then $T$ is also $\pi$-semisimple. 
Proof. Let $B$ be a $\pi$-ideal of $T$. We assume that $0 \neq x \in B$ and seek a contradiction. Clearly there exists a $\beta \in P$ satisfying the following conditions. (a) $\beta \in P$-supp $x$, and (b) if $\alpha \in P$-supp $x$, then $\alpha \ngtr \beta$. (In fact, by [7, Lemma 2] there will be more than one such $\beta$.) Let $P^{\prime}=\{\alpha \in P \mid \alpha<\gamma$ and $\gamma \in P$-supp $x\}$; then $P^{\prime}$ is a semilattice. Hence $T^{\prime}=\Sigma_{\alpha \in P^{\prime}} T_{\alpha}$ is both a supplementary semilattice sum of subrings and an ideal of $T$. Let $A=B \cap T^{\prime}$. Then $x \in A$. Since $A$ is an ideal of the $\pi$-ring $B$, then $A$ is $\pi$-ring by our standing hypothesis on $\pi$. Since $A$ is also an ideal of $T^{\prime}$, then $A$ is a $\pi$-ideal of $T^{\prime}$.

Let $\theta: T^{\prime} \rightarrow T_{\beta}$ be the projection map. Then $0 \neq \theta(x) \in \theta(A)$ by (a). Clearly $\theta$ preserves addition. Let $c_{1}, c_{2} \in T^{\prime}$, and write $c_{i}=d_{i}+e_{i}(i=1,2)$, where $d_{i} \in T_{\beta}$ and $e_{i} \in \sum_{\alpha \neq \beta} T_{\alpha}$. By (b), $\beta$ is maximal in $P^{\prime}$; hence $c_{1} c_{2}=d_{1} d_{2}+f$, where $\{P$-supp $f\} \cap T_{\beta}=\varnothing$. Thus $\theta\left(c_{1} c_{2}\right)=d_{1} d_{2}=\theta\left(c_{1}\right) \theta\left(c_{2}\right)$. That is, $\theta$ is a ring homomorphism of $T^{\prime}$ onto $T_{\beta}$. But then $\theta(A)$ is a nonzero $\pi$-ideal of $T_{\beta}$, which contradicts the hypothesis that $T_{\beta}$ is $\pi$-semisimple.

We now obtain three results needed in the proof of Theorem 2.

LEMMA 1. If $\mu$ is a zero element of $P$ and if $R[S]$ is $\pi$-semisimple, then $R\left[S_{\mu}\right]$ is $\pi$-semisimple.

Proof. Let $A$ be a $\pi$-ideal of $R\left[S_{\mu}\right]$. If $s \in R\left[S_{\alpha}\right]$ for some $\alpha$ and $a \in A$, then $s a=\left(s \varphi_{\alpha, \mu}\right) a \in A$ and $a s=a\left(s \varphi_{\alpha, \mu}\right) \in A$. It follows that $A$ is also an ideal of $R[S]$, and hence $A=0$.

Let $\alpha, \beta \in P$. Then $\beta$ is said to be maximal under $\alpha$ if $\beta<\alpha$ and there is no $\gamma \in P$ such that $\beta<\gamma<\alpha$. Further, $P$ is called an $m . u$.-semilattice if $P$ satisfies the following conditions.

(1) If $\alpha, \delta \in P$ with $\delta<\alpha$, then there exists $\beta$ maximal under $\alpha$ such that $\delta<\beta<\alpha$.

(2) The set $\{\beta \in P \mid \beta$ is maximal under $\alpha\}$ is finite for each $\alpha \in P$.

LeMma 2. Let $P$ be an m.u.-semilattice, and suppose $\alpha \in P$ is not a zero of $P$. Let $\left\{\beta_{1}, \beta_{2}, \ldots, \beta_{n}\right\}$ be the set of elements maximal under $\alpha$. For each nonempty subset $\Delta$ of $\left\{\beta_{1}, \beta_{2}, \ldots, \beta_{n}\right\}$, let $\Delta^{\prime}$ denote the product (g.l.b.) of all the $\beta_{i}$ in $\Delta$, and let $|\Delta|$ denote the cardinality of $\Delta$. If $A$ is an ideal of $R\left[S_{\alpha}\right]$, then for each $a \in A$, define

$$
\begin{aligned}
a^{*}= & a-\left[\sum_{|\Delta|=1} a \varphi_{\alpha, \Delta^{\prime}}\right]+\sum_{|\Delta|=2} a \varphi_{\alpha, \Delta^{\prime}}-\left[\sum_{|\Delta|=3} a \varphi_{\alpha, \Delta^{\prime}}\right] \\
& +\cdots+(-1)^{n} a \varphi_{\alpha, \beta_{1} \beta_{2} \cdots \beta_{n} .}
\end{aligned}
$$

Then $A^{*}=\left\{a^{*} \mid a \in A\right\}$ is an ideal of $R[S]$.

Proof. Let $a^{*} \in A^{*}$, and let $x \in R\left[S_{\gamma}\right]$ for some $\gamma \in P$. We wish to show that $x a^{*} \in A^{*}$.

Suppose that $\gamma \geqslant \alpha$. Since $\left(x \varphi_{\gamma, \alpha}\right) a \in A$, then

$$
\begin{aligned}
x a^{*}= & \left(x \varphi_{\gamma, \alpha}\right) a-\left[\sum_{|\Delta|=1}\left(\left(x \varphi_{\gamma, \alpha}\right) a\right) \varphi_{\alpha, \Delta^{\prime}}\right]+\left[\sum_{|\Delta|=2}\left(\left(x \varphi_{\gamma, \alpha}\right) a\right) \varphi_{\alpha, \Delta^{\prime}}\right]-\cdots \\
& +(-1)^{n}\left(\left(x \varphi_{\gamma, \alpha}\right) a\right) \varphi_{\alpha, \beta_{1} \beta_{2}} \cdots \beta_{n} \in A^{*}
\end{aligned}
$$


Suppose that $\gamma<\alpha$. Then, after reindexing, $\gamma<\beta_{1} \beta_{2} \cdots \beta_{k}$ for some maximal $k$, where $1<k<n$. If $\pm a \varphi_{\alpha, \Delta^{\prime}}$ is a term of $a^{*}$ and $\Delta \subseteq\left\{\beta_{1}, \beta_{2}, \ldots, \beta_{k}\right\}$, then $\pm x\left(a \varphi_{\alpha, \Delta^{\prime}}\right)= \pm x\left(a \varphi_{\alpha, \gamma}\right)$. The sum of the terms of $x a^{*}$ having this form is

$$
x\left(a \varphi_{\alpha, \gamma}\right)\left[1-k+\left(\begin{array}{l}
k \\
2
\end{array}\right)-\left(\begin{array}{l}
k \\
3
\end{array}\right)+\cdots+(-1)^{k}\right]=x\left(a \varphi_{\alpha, \gamma}\right)[1+(-1)]^{k}=0 .
$$

All other terms of $x a^{*}$ involve some $\varphi_{\alpha, \Delta^{\prime} \Gamma^{\prime}}$, where $\Delta$ is a subset of $\left\{\beta_{1}, \beta_{2}, \ldots, \beta_{k}\right\}$, $\Gamma$ is a nonempty subset of $\left\{\beta_{k+1}, \beta_{k+2}, \ldots, \beta_{n}\right\}$, and $\Gamma^{\prime}$ is the product (g.l.b.) of the elements of $\Gamma$. For a given $\Gamma$,

$$
x\left(a \varphi_{\alpha, \Delta^{\prime} \Gamma^{\prime}}\right)=\left(x \varphi_{\gamma, \gamma \Delta^{\prime} \Gamma^{\prime}}\right)\left(a \varphi_{\alpha, \gamma \Delta^{\prime} \Gamma^{\prime}}\right)=\left(x \varphi_{\gamma, \gamma \Gamma^{\prime}}\right)\left(a \varphi_{\alpha, \gamma \Gamma^{\prime}}\right)=x\left(a \varphi_{\alpha, \gamma \Gamma^{\prime}}\right)
$$

for all $\Delta$. In $x a^{*}$, the sign of the $\Delta^{\prime} \Gamma^{\prime}$ term is equal (opposite) that of the $\Gamma^{\prime}$ term if $|\Delta|$ is even (odd). Hence, for a given $\Gamma$, the sum over $\Delta$ of the $\Delta^{\prime} \Gamma^{\prime}$ terms is

$$
x\left(a \varphi_{\alpha, \gamma \Gamma^{\prime}}\right)\left[1-k+\left(\begin{array}{l}
k \\
2
\end{array}\right)-\left(\begin{array}{l}
k \\
3
\end{array}\right)+\cdots+(-1)^{k}\right]=0 .
$$

Since every term of $x a^{*}$ appears exactly once in an expression $(\dagger)$ or $(\dagger+)$, then $x a^{*}=0 \in A^{*}$.

Suppose that $\alpha$ and $\gamma$ are not related by $<$; i.e., suppose that neither $\alpha<\gamma$ nor $\alpha>\gamma$ holds. Then for all nonempty $\Delta \subseteq\left\{\beta_{1}, \beta_{2}, \ldots, \beta_{n}\right\}$,

$$
x\left(a \varphi_{\alpha, \Delta^{\prime}}\right)=\left(x \varphi_{\gamma, \gamma \Delta^{\prime}}\right)\left(a \varphi_{\alpha, \gamma \Delta^{\prime}}\right)=\left(x \varphi_{\gamma, \alpha \gamma} \varphi_{\alpha \gamma, \alpha \gamma \Delta^{\prime}}\right)\left(a \varphi_{\alpha, \alpha \gamma \Delta^{\prime}}\right)=\left(x \varphi_{\gamma, \alpha \gamma}\right)\left(a \varphi_{\alpha, \Delta^{\prime}}\right) .
$$

If $\Delta$ is the empty set, define $x\left(a \varphi_{\alpha, \Delta^{\prime}}\right)=x a$. Thus we may replace $x$ by $x \varphi_{\gamma, \alpha \gamma}$ in this case; hence it follows from the preceding paragraph that $x a^{*}=0 \in A^{*}$.

The three preceding paragraphs show that $A^{*}$ is closed under left multiplication by elements of any $S_{\gamma}$ and hence by elements of $R[S]$. A symmetric argument shows that $A^{*}$ is closed under right multiplication by elements of $R[S]$. Clearly $A^{*}$ is closed under addition. Thus $A^{*}$ is an ideal of $R[S]$.

LEMMA 3. Under the hypotheses of Lemma 2, the mapping *:A $\rightarrow A^{*}: a \rightarrow a^{*}$ is a ring isomorphism of $A$ onto $A^{*}$.

Proof. Let $a_{1}, a_{2} \in A$. Then $a_{1}{ }^{*} a_{2}{ }^{*}=a_{1} a_{2}{ }^{*}+$ terms of the form $\left(a_{1} \varphi_{\alpha, \gamma}\right) a_{2}{ }^{*}$, where $\gamma<\alpha$. It follows from the case $\gamma \geqslant \alpha$ in the proof of Lemma 2 that $a_{1} a_{2}{ }^{*}=\left(a_{1} a_{2}\right)^{*}$. It follows from the case $\gamma<\alpha$ in the proof of Lemma 2 that each of the remaining terms $\left(a_{1} \varphi_{\alpha, \gamma}\right) a_{2}{ }^{*}$ is 0 . Hence $a_{1}{ }^{*} a_{2}{ }^{*}=a_{1} a_{2}{ }^{*}=\left(a_{1} a_{2}\right)^{*}$. The formula $a_{1}^{*}+a_{2}^{*}=\left(a_{1}+a_{2}\right)^{*}$ follows from the commutativity of addition and the homomorphism properties of the $\varphi_{\alpha, \beta}$. Clearly, ${ }^{*}$ is a 1-1 map.

We are now ready for our main result.

TheOREM 2. Let $P$ be an m.u.-semilattice. Then $R[S]$ is $\pi$-semisimple if and only if $R\left[S_{\alpha}\right]$ is $\pi$-semisimple for each $\alpha \in P$.

Proof. The "if" part follows from Theorem 1.

For the "only if" part, let $A$ be a $\pi$-ideal of $R\left[S_{\alpha}\right]$ for some $\alpha$. If $\alpha$ is a zero of $P$, then $A=0$ by Lemma 1 . If $\alpha$ is not a zero of $P$, then $A^{*}=\left\{a^{*} \mid a \in A\right\}$ is a $\pi$-ideal 
of $R[S]$ by Lemma 2 and 3 ; hence we must also have $A=0$ in this case. Therefore, $R\left[S_{\alpha}\right]$ is $\pi$-semisimple.

Question 1 [7, p. 477] is as follows: Find a condition on $P$ such that if $T$ is a supplementary semilattice sum of $T_{\alpha}(\alpha \in P)$, then $T \pi$-semisimple implies $T_{\alpha}$ is $\pi$-semisimple for each $\alpha \in P$. Theorem 2 states that $P$ being an m.u.-semilattice is such a condition when $T=R[S]$.

Corollary 1. Let $P$ be an m.u.-semilattice. Then $R[S]$ is semiprime if and only if $R\left[S_{\alpha}\right]$ is semiprime for each $\alpha \in P$.

COROllary 2. Let $P$ be an m.u.-semilattice. Then $R[S]$ is nil semisimple if and only if $R\left[S_{\alpha}\right]$ is nil semisimple for each $\alpha \in P$.

Corollary 3. Let $P$ be an m.u.-semilattice. Then $R[S]$ is Jacobson semisimple if and only if $R\left[S_{\alpha}\right]$ is Jacobson semisimple for each $\alpha \in P$.

The condition that $P$ be an m.u.-semilattice cannot be dropped from the hypothesis of Theorem 2 . In fact, it is possible for $R[S]$ to be Jacobson semisimple and for some $R\left[S_{\alpha}\right]$ to have a nonzero nilpotent ideal if either of the two conditions in the definition of m.u.-semilattice is dropped. Example 1 (Example 2) shows that condition (1) (condition (2)) in the definition of m.u.-semilattice cannot be dropped. Both examples answer in the negative Question 7 of Weissglass [7, p. 477].

The support of $x=\Sigma_{s \in S} r_{s} \in R[S]$, denoted by supp $x$, is the set $\left\{s \in S \mid r_{s} \neq\right.$ $0\}$.

EXAMPLE 1. Let $R$ be a field of characteristic $p>0$, let $Z_{p}$ be the cyclic group of $p$ elements, and let $C$ denote the complex numbers. Let $P=[0, \omega]$, the initial ordinal segment terminating at the first infinite ordinal $\omega$. For $0<n<\omega$, let $S_{n}=Z_{p} \sim C$ (wreath product); define $S_{\omega}$ to be a direct sum of $C$ copies of $Z_{p}$. We use the following maps $\varphi_{\alpha, \beta}$ to define $S=\cup_{\alpha<\omega} S_{\alpha} S_{\alpha}$ as a semilattice $P$ of semigroups: $\varphi_{\alpha, \beta}$ is the identity map on $Z_{p} \imath C$ for $0<\beta<\alpha<\omega$, and $\varphi_{\omega, \beta}$ is the natural embedding of $S_{\omega}$ as a normal subgroup of $S_{\beta}=Z_{p} \downarrow C$ for $0<\beta<\omega$. From [4, Lemma VII.4.12] it follows that $R\left[S_{\alpha}\right]$ is Jacobson semisimple for $0<\alpha<\omega$. Since $S_{\omega}$ is an Abelian $p$-group, then $R\left[S_{\omega}\right]$ has a nonzero nilpotent ideal by [4, Theorem IV.2.13]. Let $K=\Sigma_{\alpha<\omega} R\left[S_{\alpha}\right.$ ]. Then $K$ is an ideal of $R[S]$; moreover, $K$ is a Jacobson semisimple ring by Theorem 1. If $N$ is a quasi-regular ideal of $R[S]$, then $N \cap K=0$. Thus, if $0 \neq x \in N$, then $\{\operatorname{supp} x\} \cap S_{\omega} \neq \varnothing$. If $\{\operatorname{supp} x\} \cap S_{\alpha} \neq \varnothing$ for some $\alpha<\omega$, let $m=1+\max \{\alpha \mid 0<\alpha<\omega$ and $\{\operatorname{supp} x\}$ $\left.\cap S_{\alpha} \neq \varnothing\right\}$; otherwise, let $m=1$. Let $e_{m}$ denote the identity element of $S_{m}$. Then $\left\{\right.$ supp $\left.e_{m} x\right\} \cap S_{m} \neq \varnothing$ and $e_{m} x \in K \cap N$, which contradicts the fact that $K \cap N$ $=0$. Hence $R[S]$ is a Jacobson semisimple ring.

EXAMPLe 2. Let $P=[0, \omega]$ as a set, but impose a new order $\gg$ on $P$ (rather than the usual order $>$ ) by the following rules: (i) if $m, n, \omega$ are distinct elements of $P$, then $m \wedge n=0$ in the order $\gg$; (ii) if $m \neq \omega$, then $\omega \gg m$. Thus $P$ can be made into a semilattice with the following order structure. 


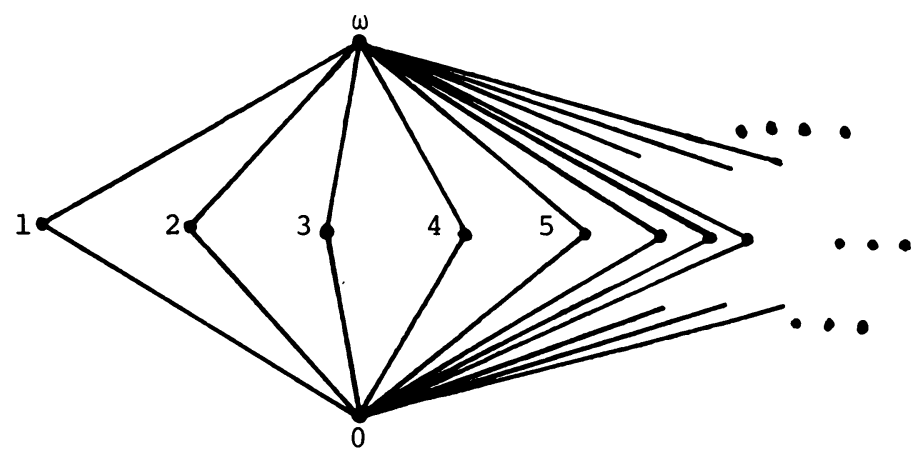

Now let $R, S_{\alpha}, \varphi_{\alpha, \beta}, S, K$, and $N$ be defined as in Example 1, except that $\gg$ replaces $>$ in the definition of these terms. Thus $R\left[S_{\alpha}\right]$ is Jacobson semisimple whenever $\omega \gg \alpha, R\left[S_{\omega}\right]$ has a nonzero nilpotent ideal, and $K \cap N=0$ for any quasi-regular ideal $N$ of $R[S]$. Thus if $0 \neq x \in N$, then $\{\operatorname{supp} x\} \cap S_{\omega} \neq \varnothing$. Since $\{\operatorname{supp} x\}$ is finite, there exists $m \in P-\{0, \omega\}$ such that $\{\operatorname{supp} x\} \cap S_{m}=\varnothing$. Let $e_{m}$ denote the identity element of $S_{m}$. Then $\left\{\operatorname{supp} e_{m} x\right\} \cap S_{m} \neq \varnothing$ and $e_{m} x \in K \cap$ $N$, which contradicts the fact that $K \cap N=0$. Hence $R[S]$ is a Jacobson semisimple.

J. Weissglass has asked the following question [7, Question 2, p. 477]. If $T=\Sigma_{\alpha \in \Omega} T_{\alpha}$ is a supplementary semilattice sum and $T$ is commutative, does $T$ Jacobson semisimple imply that each $T_{\alpha}$ is Jacobson semisimple? We apply our methods to answer this question in the negative in the following example.

Example 3. Let $P$ be the semilattice of Example 1. Let $T_{\omega}$ be a commutative local integral domain with identity element that is not a field. Let $T_{n}$ be the quotient field of $T_{\omega}$ for $0 \leqslant n<\omega$. Define the homomorphisms $\varphi_{\alpha, \beta}$ to be the identity map on $T_{n}$ for $0 \leqslant \beta \leqslant \alpha<\omega$; define $\varphi_{\omega, \beta}$ to be the natural embedding of $T_{\omega}$ into $T_{\beta}$ for each $\beta \leqslant \omega$. Then $T=\Sigma_{\beta<\omega} T_{\beta}$ forms a commutative supplementary semilattice sum of subrings $T_{\beta}$ by naturally extending the multiplication $x \cdot y=\varphi_{\alpha, \alpha \wedge \beta}(x) \cdot \varphi_{\beta, \alpha \wedge \beta}(y)$ for $x \in T_{\alpha}, y \in T_{\beta}$. Now $T_{\omega}$ is not Jacobson semisimple. Since $T_{\beta}$ is Jacobson semisimple for each $\beta<\omega$, then $K=\Sigma_{\beta<\omega} T_{\beta}$ is Jacobson semisimple by Theorem 1. If $N$ is a quasi-regular ideal of $T$, then $K \cap N=0$. If $0 \neq x \in N$, then $\omega \in P$-supp $x$. If $\alpha \in\{P$-supp $x\} \neq \varnothing$ for some $\alpha<\omega$, let $m=1+\max \{\alpha \mid 0<\alpha<\omega$ and $\alpha \in\{P$-supp $x\}\}$; otherwise, let $m=1$. Let $e_{m}$ denote the identity element of $T_{m}$. Then $m \in P$-supp $e_{m} x$ and $e_{m} x \in K \cap$ $N$, which contradicts the fact that $K \cap N=0$. Hence $T$ is a Jacobson semisimple ring.

\section{REFERENCES}

1. A. H. Clifford and G. B. Preston, Algebraic theory of semgroups, Vol. I, Math. Surveys, No. 7, Amer. Math. Soc., Providence, R. I., 1961; reprinted 1964.

2. R. Gilmer and T. Parker, Nilpotent elements of commutative semigroup rings, Michigan Math. J. 22 (1975), 97-108.

3. J. Krempa, On semigroup rings. III, Bull. Acad. Polon. Sci. Sér. Sci. Math. Astronom. Phys. 25 (1977), 225-231. 
4. D. S. Passman, The algebraic structure of group rings, Wiley, New York, 1977.

5. M. Petrich, Introduction to semigroups, Merrill, Columbus, Ohio, 1973.

6. A. Quesada, Properties of twisted semigroup rings, Thesis, Univ. of Florida, 1978.

7. J. Weissglass, Semigroup rings and semilattice suons of rings, Proc. Amer. Math. Soc. 39 (1973), 471-478.

Departmient of Mathematics, University of Florida, Gannesville, Florida 32611 (Current address of M. L. Teply and E. G. Turman)

Current address (Antonio Quesada): Department of Mathematics, The Catholic University of Puerto Rico, Ponce, Puerto Rico 00731 